hep-ph/0001005

CERN-TH/2K-001

UMN-TH-1834/2K

TPI-MINN-2K/01

\title{
Re-Evaluation of the Elastic Scattering of Supersymmetric Dark Matter
}

\author{
John Ellis ${ }^{1}$, Andrew Ferstl ${ }^{2}$ and Keith A. Olive ${ }^{2,3}$ \\ ${ }^{1}$ TH Division, CERN, Geneva, Switzerland \\ ${ }^{2}$ School of Physics and Astronomy, University of Minnesota, Minneapolis, MN 55455, USA \\ ${ }^{3}$ Theoretical Physics Institute, University of Minnesota, Minneapolis, MN 55455, USA
}

\begin{abstract}
We examine the cross sections for the elastic scattering of neutralinos $\chi$ on nucleons $p, n$, as functions of $m_{\chi}$ in the constrained minimal supersymmetric standard model. We find narrow bands of possible values of the cross section, that are considerably lower than some previous estimates. The constrained model is based on the minimal supergravity-inspired framework for the MSSM, with universal scalar and gaugino masses $m_{0}, m_{1 / 2}$, and $\mu$ and the MSSM Higgs masses treated as dependent parameters. We explore systematically the region of the $\left(m_{1 / 2}, m_{0}\right)$ plane where LEP and other accelerator constraints are respected, and the relic neutralino density lies in the range $0.1 \leq \Omega_{\chi} h^{2} \leq 0.3$ preferred by cosmology. We update previous discussions of both the spin-independent and -dependent scattering matrix elements on protons and neutrons, using recent analyses of low-energy hadron experiments.
\end{abstract}

CERN-TH/2K-001

January 2000 


\section{Introduction}

One of the most promising candidates for the cold dark matter believed to pervade the Universe is the lightest supersymmetric particle (LSP) [1], commonly expected to be the lightest neutralino $\chi$, which is stable in the minimal supersymmetric extension of the Standard Model (MSSM) with conserved $R$ parity [2]. The quantum stability of the gauge hierarchy suggests that sparticles weigh less than about $1 \mathrm{TeV}$ [3], which is also the range favoured for a cold dark matter particle [4], and there are indeed generic domains of the MSSM parameter space in which the relic LSP density falls within the range $0.1 \leq \Omega_{\chi} h^{2} \leq 0.3$ favoured by astrophysics and cosmology [1]. The unsuccessful laboratory searches for sparticles impose non-trivial constraints on the MSSM parameter space, suggesting that the LSP $\chi$ is mainly a $U(1)$ gaugino (Bino) [5].

Many non-accelerator strategies to search for cosmological relic neutralinos have been proposed [6], including indirect searches for products of their annihilations in free space or inside astrophysical bodies, and direct searches for their scattering on target nuclei in low-background underground laboratories [7]. The rates for such experiments typically have larger uncertainties than those for producing sparticles at accelerators, since they involve some astrophysical and/or cosmological uncertainties as well as those due to simulations of the signatures, over and above the common uncertainties in the MSSM parameters. Nevertheless, such dark matter searches offer interesting prospects for beating accelerators to

the discovery of supersymmetry, particularly during the coming years before the LHC enters operation.

In this paper, we embark on a programme to clarify the extents of the uncertainties in searches for supersymmetric dark matter, by re-evaluating the rates to be expected for the elastic scattering of relic LSPs on protons and neutrons [8, 9]. Large ranges for these rates are often quoted [10], reflecting general explorations of the MSSM parameter space. Our approach is to establish as accurately as possible a baseline set of predictions based on the most plausible assumptions commonly used in constrained MSSM phenomenology, such as universality in the soft supersymmetry-breaking parameters as suggested by minimal supergravity models, and requiring the cosmological relic density to lie within the range favoured by astrophysics and cosmology, namely $0.1 \leq \Omega_{\chi} h^{2} \leq 0.3$. These assumptions can and should be questioned, but they are well motivated and good candidates for default options in analyses of the MSSM and cold dark matter.

In the course of this re-evaluation of elastic $\chi-p, n$ scattering cross sections, we reanalyze the relevant spin-independent and spin-dependent matrix elements of scalar densities 
and axial currents in protons and neutrons. We update previous analyses using further information from chiral symmetry [11, 12], low-energy $\pi-p, n$ scattering [13 and deepinelastic lepton-nucleon scattering [14]. We include a discussion of uncertainties in the values of the scalar and axial-current matrix elements.

We perform a systematic scan of the region of the $m_{0}, m_{1 / 2}$ parameter space of the MSSM with supergravity-inspired universality that is consistent with accelerator constraints and yields a cosmological relic density within the favoured range $0.1 \leq \Omega_{\chi} \leq 0.3$ [15]. We treat $\mu$ as a dependent parameter (modulo a sign ambiguity), and our results are not very sensitive to $A$. We order our results in terms of $m_{\chi}$ which closely tracks $m_{1 / 2}$. For any given choice of $m_{\chi}, \tan \beta$ and the sign of $\mu$, we find a relatively narrow band of possible cross sections, reflecting the fact that the accelerator and cosmological constraints [5, 15] favour a predominant $U(1)$ gaugino (Bino) composition for the LSP. Our results fall considerably below many of the possible predictions in the literature [10], and may discourage some fainthearted experimentalists. However, we think they provide a realistic estimate of the target sensitivity required for an experiment to have a good chance of success.

\section{Theoretical Framework}

We review in this Section the theoretical framework we use in the context of the MSSM [2]. The neutralino LSP is the lowest-mass eigenstate combination of the Bino $\tilde{B}$, Wino $\tilde{W}$ and Higgsinos $\tilde{H}_{1,2}$, whose mass matrix $N$ is diagonalized by a matrix $Z$ : $\operatorname{diag}\left(m_{\chi_{1}, ., 4}\right)=$ $Z^{*} N Z^{-1}$. The composition of the lightest neutralino may be written as

$$
\chi=Z_{\chi 1} \tilde{B}+Z_{\chi 2} \tilde{W}+Z_{\chi 3} \tilde{H}_{1}+Z_{\chi 4} \tilde{H}_{2}
$$

As already mentioned, we assume universality at the supersymmetric GUT scale for the

$U(1)$ and $S U(2)$ gaugino masses: $M_{1,2}=m_{1 / 2}$, so that $M_{1}=\frac{5}{3} \tan ^{2} \theta_{W} M_{2}$ at the electroweak scale. We denote by $\tan \beta$ the ratio of Higgs vacuum expectation values, and $\mu$ is the Higgsino mass-mixing parameter. We also assume GUT-scale universality for the soft supersymmetrybreaking scalar masses $m_{0}$, for the Higgs bosons as well as the squarks and sleptons. We further assume GUT-scale universality for the soft supersymmetry-breaking trilinear terms A. Our treatment of the sfermion mass matrices $M$ follows [16]. As discussed there, the sfermion mass-squared matrix is diagonalized by a matrix $\eta$ : $\operatorname{diag}\left(m_{1}^{2}, m_{2}^{2}\right) \equiv \eta M^{2} \eta^{-1}$, which can be parameterized for each flavour $f$ by an angle $\theta_{f}$ and phase $\gamma_{f}$ :

$$
\left(\begin{array}{cc}
\cos \theta_{f} & \sin \theta_{f} e^{i \gamma_{f}} \\
-\sin \theta_{f} e^{-i \gamma_{f}} & \cos \theta_{f}
\end{array}\right) \equiv\left(\begin{array}{ll}
\eta_{11} & \eta_{12} \\
\eta_{21} & \eta_{22}
\end{array}\right)
$$


As a simplification, we neglect $\mathrm{CP}$ violation in this paper, so that $\gamma_{f}=0$ and there are no $\mathrm{CP}$-violating phases in the neutralino mass matrix, either. We treat $m_{1 / 2}, m_{0}, A$ and $\tan \beta$ as free parameters, and $\mu$ and the pseudoscalar Higgs mass $m_{A}$ as dependent parameters specified by the electroweak vacuum conditions, which we calculate using $m_{t}=175 \mathrm{GeV}$ 日.

The MSSM Lagrangian leads to the following low-energy effective four-fermi Lagrangian suitable for describing elastic $\chi$-nucleon scattering [16]:

$$
\mathcal{L}=\bar{\chi} \gamma^{\mu} \gamma^{5} \chi \bar{q}_{i} \gamma_{\mu}\left(\alpha_{1 i}+\alpha_{2 i} \gamma^{5}\right) q_{i}+\alpha_{3 i} \bar{\chi} \chi \bar{q}_{i} q_{i}+\alpha_{4 i} \bar{\chi} \gamma^{5} \chi \bar{q}_{i} \gamma^{5} q_{i}+\alpha_{5 i} \bar{\chi} \chi \bar{q}_{i} \gamma^{5} q_{i}+\alpha_{6 i} \bar{\chi} \gamma^{5} \chi \bar{q}_{i} q_{i}
$$

This Lagrangian is to be summed over the quark generations, and the subscript $i$ labels uptype quarks $(i=1)$ and down-type quarks $(i=2)$. The terms with coefficients $\alpha_{1 i}, \alpha_{4 i}, \alpha_{5 i}$ and $\alpha_{6 i}$ make contributions to the elastic scattering cross section that are velocity-dependent, and may be neglected for our purposes. In fact, if the CP violating phases are absent as assumed here, $\alpha_{5}=\alpha_{6}=0$ [17]. The coefficients relevant for our discussion are:

$$
\begin{aligned}
\alpha_{2 i}= & \frac{1}{4\left(m_{1 i}^{2}-m_{\chi}^{2}\right)}\left[\left|Y_{i}\right|^{2}+\left|X_{i}\right|^{2}\right]+\frac{1}{4\left(m_{2 i}^{2}-m_{\chi}^{2}\right)}\left[\left|V_{i}\right|^{2}+\left|W_{i}\right|^{2}\right] \\
& -\frac{g^{2}}{4 m_{Z}^{2} \cos ^{2} \theta_{W}}\left[\left|Z_{\chi_{3}}\right|^{2}-\left|Z_{\chi_{4}}\right|^{2}\right] \frac{T_{3 i}}{2}
\end{aligned}
$$

and

$$
\begin{aligned}
\alpha_{3 i}= & -\frac{1}{2\left(m_{1 i}^{2}-m_{\chi}^{2}\right)} \operatorname{Re}\left[\left(X_{i}\right)\left(Y_{i}\right)^{*}\right]-\frac{1}{2\left(m_{2 i}^{2}-m_{\chi}^{2}\right)} \operatorname{Re}\left[\left(W_{i}\right)\left(V_{i}\right)^{*}\right] \\
& -\frac{g m_{q i}}{4 m_{W} B_{i}}\left[\operatorname{Re}\left(\delta_{1 i}\left[g Z_{\chi 2}-g^{\prime} Z_{\chi 1}\right]\right) D_{i} C_{i}\left(-\frac{1}{m_{H_{1}}^{2}}+\frac{1}{m_{H_{2}}^{2}}\right)\right. \\
& \left.+\operatorname{Re}\left(\delta_{2 i}\left[g Z_{\chi 2}-g^{\prime} Z_{\chi 1}\right]\right)\left(\frac{D_{i}^{2}}{m_{H_{2}}^{2}}+\frac{C_{i}^{2}}{m_{H_{1}}^{2}}\right)\right]
\end{aligned}
$$

where

$$
\begin{aligned}
X_{i} & \equiv \eta_{11}^{*} \frac{g m_{q_{i}} Z_{\chi 5-i}^{*}}{2 m_{W} B_{i}}-\eta_{12}^{*} e_{i} g^{\prime} Z_{\chi 1}^{*} \\
Y_{i} & \equiv \eta_{11}^{*}\left(\frac{y_{i}}{2} g^{\prime} Z_{\chi 1}+g T_{3 i} Z_{\chi 2}\right)+\eta_{12}^{*} \frac{g m_{q_{i}} Z_{\chi 5-i}}{2 m_{W} B_{i}} \\
W_{i} & \equiv \eta_{21}^{*} \frac{g m_{q_{i}} Z_{\chi 5-i}^{*}}{2 m_{W} B_{i}}-\eta_{22}^{*} e_{i} g^{\prime} Z_{\chi 1}^{*} \\
V_{i} & \equiv \eta_{22}^{*} \frac{g m_{q_{i}} Z_{\chi 5-i}}{2 m_{W} B_{i}}+\eta_{21}^{*}\left(\frac{y_{i}}{2} g^{\prime} Z_{\chi 1}+g T_{3 i} Z_{\chi 2}\right)
\end{aligned}
$$

\footnotetext{
${ }^{1}$ We have checked that varying $m_{t}$ by $\pm 5 \mathrm{GeV}$ has a negligible effect on our results.
} 
where $y_{i}, T_{3 i}$ denote hypercharge and isospin, and

$$
\begin{array}{cl}
\delta_{1 i}=Z_{\chi 3}\left(Z_{\chi 4}\right) \quad, \quad \delta_{2 i}=Z_{\chi 4}\left(-Z_{\chi 3}\right), \\
B_{i}=\sin \beta(\cos \beta) \quad, \quad A_{i}=\cos \beta(-\sin \beta), \\
C_{i}=\sin \alpha(\cos \alpha) \quad, \quad D_{i}=\cos \alpha(-\sin \alpha)
\end{array}
$$

for up (down) type quarks. We denote by $m_{H_{2}}<m_{H_{1}}$ the two scalar Higgs masses, and $\alpha$ denotes the Higgs mixing angle ?f

\section{$3 \quad$ Hadronic Matrix Elements}

The elastic cross section for scattering off a nucleus can be decomposed into a scalar (spinindependent) part obtained from the $\alpha_{2 i}$ term in (3), and a spin-dependent part obtained from the $\alpha_{3 i}$ term. Each of these can be written in terms of the cross sections for elastic scattering for scattering off individual nucleons, as we now review and re-evaluate.

The scalar part of the cross section can be written as

$$
\sigma_{3}=\frac{4 m_{r}^{2}}{\pi}\left[Z f_{p}+(A-Z) f_{n}\right]^{2}
$$

where $m_{r}$ is the reduced LSP mass,

$$
\frac{f_{p}}{m_{p}}=\sum_{q=u, d, s} f_{T q}^{(p)} \frac{\alpha_{3 q}}{m_{q}}+\frac{2}{27} f_{T G}^{(p)} \sum_{c, b, t} \frac{\alpha_{3 q}}{m_{q}}
$$

and $f_{n}$ has a similar expression. The parameters $f_{T q}^{(p)}$ are defined by

$$
m_{p} f_{T q}^{(p)} \equiv\left\langle p\left|m_{q} \bar{q} q\right| p\right\rangle \equiv m_{q} B_{q}
$$

whilst $f_{T G}^{(p)}=1-\sum_{q=u, d, s} f_{T q}^{(p)}$ [19]. We observe that only the products $m_{q} B_{q}$, the ratios of the quark masses $m_{q}$ and the ratios of the scalar matrix elements $B_{q}$ are invariant under renormalization and hence physical quantities.

We take the ratios of the quark masses from [1]:

$$
\frac{m_{u}}{m_{d}}=0.553 \pm 0.043, \quad \frac{m_{s}}{m_{d}}=18.9 \pm 0.8
$$

In order to determine the ratios of the $B_{q}$ and the products $m_{q} B_{q}$ we use information from chiral symmetry applied to baryons. Following [12], we have:

$$
z \equiv \frac{B_{u}-B_{s}}{B_{d}-B_{s}}=\frac{m_{\Xi^{0}}+m_{\Xi^{-}}-m_{p}-m_{n}}{m_{\Sigma^{+}}+m_{\Sigma^{-}}-m_{p}-m_{n}}
$$

\footnotetext{
${ }^{2}$ We note that (5) is taken from [17] and corrects an error in [16], and that (4, 旬) agree with [6, 8] and the published version of [18].
} 
Substituting the experimental values of these baryon masses, we find

$$
z=1.49
$$

with an experimental error that is negligible compared with others discussed below. Defining

$$
y \equiv \frac{2 B_{s}}{B_{d}+B_{u}}
$$

we then have

$$
\frac{B_{d}}{B_{u}}=\frac{2+((z-1) \times y)}{2 \times z-((z-1) \times y)}
$$

The experimental value of the $\pi$-nucleon $\sigma$ term is [13]:

$$
\sigma \equiv \frac{1}{2}\left(m_{u}+m_{d}\right) \times\left(B_{d}+B_{u}\right)=45 \pm 8 \mathrm{MeV}
$$

and octet baryon mass differences may be used to estimate that [13]

$$
\sigma=\frac{\sigma_{0}}{(1-y)}: \quad \sigma_{0}=36 \pm 7 \mathrm{MeV}
$$

Comparing (16) and (17), we find a central value of $y=0.2$, to which we assign an error \pm 0.1 , which yields

$$
\frac{B_{d}}{B_{u}}=0.73 \pm 0.02
$$

The formal error in $y$ derived from (16) and (17) is actually \pm 0.2 , which would double the error in $B_{d} / B_{u}$. We have chosen the smaller uncertainty because we consider a value of y in excess of $30 \%$ rather unlikely. However, we do illustrate later by one example the potential consequences of a larger error in $y$.

The numerical magnitudes of the individual renormalization-invariant products $m_{q} B_{q}$ and hence the $f_{T q}^{(p)}$ may now be determined:

$$
f_{T u}^{(p)}=0.020 \pm 0.004, \quad f_{T d}^{(p)}=0.026 \pm 0.005, \quad f_{T s}^{(p)}=0.118 \pm 0.062
$$

where essentially all the error in $f_{T s}^{(p)}$ arises from the uncertainty in $y$. The corresponding values for the neutron are

$$
f_{\text {Tu }}^{(n)}=0.014 \pm 0.003, \quad f_{T d}^{(n)}=0.036 \pm 0.008, \quad f_{T s}^{(n)}=0.118 \pm 0.062
$$

It is clear already that the difference between the scalar parts of the cross sections for scattering off protons and neutrons must be rather small. 
The spin-dependent part of the elastic $\chi$-nucleus cross section can be written as

$$
\sigma_{2}=\frac{32}{\pi} G_{F}^{2} m_{r}^{2} \Lambda^{2} J(J+1)
$$

where $m_{r}$ is again the reduced neutralino mass, $J$ is the spin of the nucleus, and

$$
\Lambda \equiv \frac{1}{J}\left(a_{p}\left\langle S_{p}\right\rangle+a_{n}\left\langle S_{n}\right\rangle\right)
$$

where

$$
a_{p}=\sum_{i} \frac{\alpha_{2 i}}{\sqrt{2} G_{f}} \Delta_{i}^{(p)}, a_{n}=\sum_{i} \frac{\alpha_{2 i}}{\sqrt{2} G_{f}} \Delta_{i}^{(n)}
$$

The factors $\Delta_{i}^{(p, n)}$ parametrize the quark spin content of the nucleon. A recent global analysis of QCD sum rules for the $g_{1}$ structure functions [14], including $\mathcal{O}\left(\alpha_{s}^{3}\right)$ corrections, corresponds formally to the values

$$
\Delta_{u}^{(p)}=0.78 \pm 0.02, \quad \Delta_{d}^{(p)}=-0.48 \pm 0.02, \quad \Delta_{s}^{(p)}=-0.15 \pm 0.02
$$

whilst perturbative QCD fits to the data for $g_{1}$ tend to give broader ranges [14. In our numerical analysis, we double the formal errors in (24) to \pm 0.04 , essentially $100 \%$ correlated for the three quark flavours. In the case of the neutron, we have $\Delta_{u}^{(n)}=\Delta_{d}^{(p)}, \Delta_{d}^{(n)}=\Delta_{u}^{(p)}$, and $\Delta_{s}^{(n)}=\Delta_{s}^{(p)}$.

\section{Cosmological and Experimental Constraints}

The domain of MSSM parameter space that we explore in this paper is that defined in [15]. Several convergent measures of cosmological parameters [20] suggest that the cold dark matter density $\Omega_{C D M}=0.3 \pm 0.1$ and that the Hubble expansion rate $H \equiv h \times 100 \mathrm{~km} / \mathrm{s} / \mathrm{Mpc}$ : $h=0.7 \pm 0.1$, leading to the preferred range $0.1 \leq \Omega_{C D M} h^{2} \leq 0.3$. The upper limit on $\Omega_{C D M}$ can be translated directly into the corresponding upper limit on $\Omega_{\chi}$. However, it is possible that there is more than one component in the cold dark matter, so that $\Omega_{\chi}<\Omega_{C D M}$, opening up the possibility that $\Omega_{\chi}<0.1$. Although the MSSM parameters which lead to $\Omega_{\chi}<0.1$ tend to give larger elastic scattering cross sections, the detection rate also must be reduced because of the corresponding reduction in the density of LSPs in the Galactic halo. Here we shall neglect this possibility, assuming instead that essentially all the cold dark matter is composed of LSPs, so that $\Omega_{\chi} \geq 0.1$.

For the calculation of the relic LSP density, we follow 15], where coannihilations between $\chi$ and the sleptons $\tilde{\ell}$, particularly the lighter stau $\tilde{\tau}_{1}$, were shown to play an important role. As we discuss in more detail later, $m_{\chi}$ depends essentially on $m_{1 / 2}$, and coannihilation increases 
by a factor $\sim 2$ the cosmological upper limit on $m_{1 / 2}$ to $\sim 1400 \mathrm{GeV}$, allowing $m_{\chi} \lesssim 600 \mathrm{GeV}$. At this upper limit on $m_{1 / 2}$, there is a unique allowed value of $m_{0} \sim 350 \mathrm{GeV}$, but for lower values of $m_{1 / 2}$ the width of the allowed range of $m_{0}$ expands, reaching $50 \lesssim m_{0} \lesssim 150 \mathrm{GeV}$ when $m_{1 / 2} \sim 200 \mathrm{GeV}$. At this value of $m_{1 / 2}$ and scanning across the cosmological range in $m_{0}$, we find that $m_{\chi} \sim 80 \mathrm{GeV}$, with a small variation by $\sim 0.4 \mathrm{GeV}$. These numbers are not very sensitive to $\tan \beta$ in the range from 3 to 10 studied in 15 and here, nor are they very sensitive to the chosen value of $A$.

The lower limit on $m_{1 / 2}$ and hence $m_{\chi}$ depends on the sparticle search limits provided by LEP [5]. The most essential of these for our current purposes are those provided by the experimental lower limits on the lighter chargino mass $m_{\chi^{ \pm}}$and the lighter scalar Higgs

mass $m_{H_{2}}$. A lower limit $m_{\chi^{ \pm}} \geq 95 \mathrm{GeV}$ was assumed in 15]: unsuccessful chargino searches during higher-energy runs of LEP have now increased this lower limit to $m_{\chi^{ \pm}} \geq 100 \mathrm{GeV}$ [21], which does not reduce very much the range allowed in 15.

The impact of the recently-improved lower limits on the Higgs mass [21] is potentially more significant, particularly for $\tan \beta=3$, as displayed in Figs. 6 and 7 of [15. The present experimental lower limit for $\tan \beta=3$ is probably $m_{H_{2}}>105$ to $109 \mathrm{GeV}$ [21]. The $m_{H_{2}}$ contours shown in Figs. 6a,b and $7 \mathrm{~b}$ of [15] were not calculated with the most recent two-loop MSSM code [22], so we take the $m_{H_{2}}=100 \mathrm{GeV}$ lines in [15] as indicative constraints. These correspond to $m_{1 / 2} \sim 340(720) \mathrm{GeV}$ for $\mu>(<) 0$, corresponding in turn to $m_{\chi} \gtrsim 140(310) \mathrm{GeV}$. On the other hand, for $\tan \beta=10$, the LEP lower limit on $m_{H_{2}}$

is considerably weaker than $100 \mathrm{GeV}$ [21], and hence does not constrain significantly the allowed parameter space, as seen in Figs. 6c,d and 7c of [15].

We note in passing that requiring our present electroweak vacuum to be stable against transitions to a lower-energy state in which electromagnetic charge and colour are broken (CCB) 23 would divide the parameter regions allowed in 15 into two parts: one at large $m_{1 / 2}$ and the other at small $m_{1 / 2}$ and relatively large $m_{0}$. We do not implement the CCB constraint in our analysis, since it may be considered optional. Nor do we implement any constraint due to the observed rate of $b \rightarrow s \gamma$ decay [24], but it is well known that this reduces very substantially the parameter space allowed for $\mu<0$.

\section{Results}

As discussed above, we scan the cosmologically preferred set of parameters which yield $0.1 \leq \Omega_{\chi} h^{2} \leq 0.3$ and are consistent with the recent LEP accelerator bounds. For each value of $\tan \beta$ and sign of $\mu$, we vary $m_{1 / 2}$ and $m_{0}$ over all the allowed range. As default, we 
choose $A_{0}=-m_{1 / 2}$ in most of our computations. Then, using the hadronic inputs described in section 3, we compute separately the spin-dependent and scalar contributions from the $\alpha_{2}$ and $\alpha_{3}$ coefficients, respectively, to the elastic scattering of LSPs on both protons and neutrons.

In Figure 1, we show the resulting spin-dependent elastic cross section as a function of the LSP mass, $m_{\chi}$. Although it is barely discernible, the thicknesses of the central curves in the panels show the ranges in the cross section for fixed $m_{\chi}$ that are induced by varying $m_{0}$. At large $m_{\chi}$ where coannihilations are important, the range in the allowed values of $m_{0}$ is small and particularly little variation in the cross section is expected. The shaded regions in this and the following figures show the effects of the uncertainties in the input values of the $\Delta_{i}^{(p)}$ (24). In Figure 1a, for $\tan \beta=3, \mu<0$, we see at small $m_{\chi}$ the effect of a cancellation induced by the difference in signs between $\Delta_{u}$ and $\Delta_{d, s}$. Cancellations are possible for the other values of $\tan \beta$ and sign of $\mu$, but not in the preferred range of $m_{1 / 2}$ and $m_{0}$ used here. Aside from the cancellation, the spin-dependent cross section peaks at about $10^{-4} \mathrm{pb}$ and drops rapidly as $m_{\chi}$ increases.

In Figure 2, we show the corresponding result for the scalar cross section, based on $\alpha_{3}$. As in Figure 1, the thickness of the central curve reflects the range in $m_{0}$ sampled. The shaded region now corresponds to the uncertainties in the inputs given in (19). The scalar cross section is, in general, more sensitive to the sign of $\mu$ than is the spin-dependent cross section. Notice that, in Figure $2 \mathrm{c}$ for $\tan \beta=10$ and $\mu<0$, there is another cancellation. In this case, Higgs exchange is dominant in $\alpha_{3}$. We first note that, for $\mu<0$, both $Z_{\chi 3}$ and $Z_{\chi 4}$ are negative, as is the Higgs mixing angle $\alpha$. Inserting the definitions of $\delta_{1 i(2 i)}$, we see that there is a potential cancellation of the Higgs contribution to $\alpha_{3}$ for both up-type and down-type quarks. Whilst there is such a cancellation for the down-type terms, which change from positive to negative as one increases $m_{\chi}$, such a cancellation does not occur for the up-type terms, which remain negative in the region of parameters we consider. The cancellation that is apparent in the figure is due to the cancellation in $\alpha_{3}$ between the up-type contribution (which is negative) and the down-type contribution, which is initially positive but decreasing, eventually becoming negative as we increase $m_{\chi}$.

In Figure 3, we show the effects of varying some of the input assumptions made earlier. For example, when the assumed uncertainty in $y$ is taken to be \pm 0.2 , we get a thicker shaded region, as shown for $\tan \beta=3, \mu<0$ in Figure 3a. In Figure 3b, we give one example of the cross section for the elastic scattering of neutralinos on neutrons. This particular case was chosen because it displays the largest difference between the neutron and proton cross sections among those tested. As one can see, our results for the LSP scattering on neutrons 

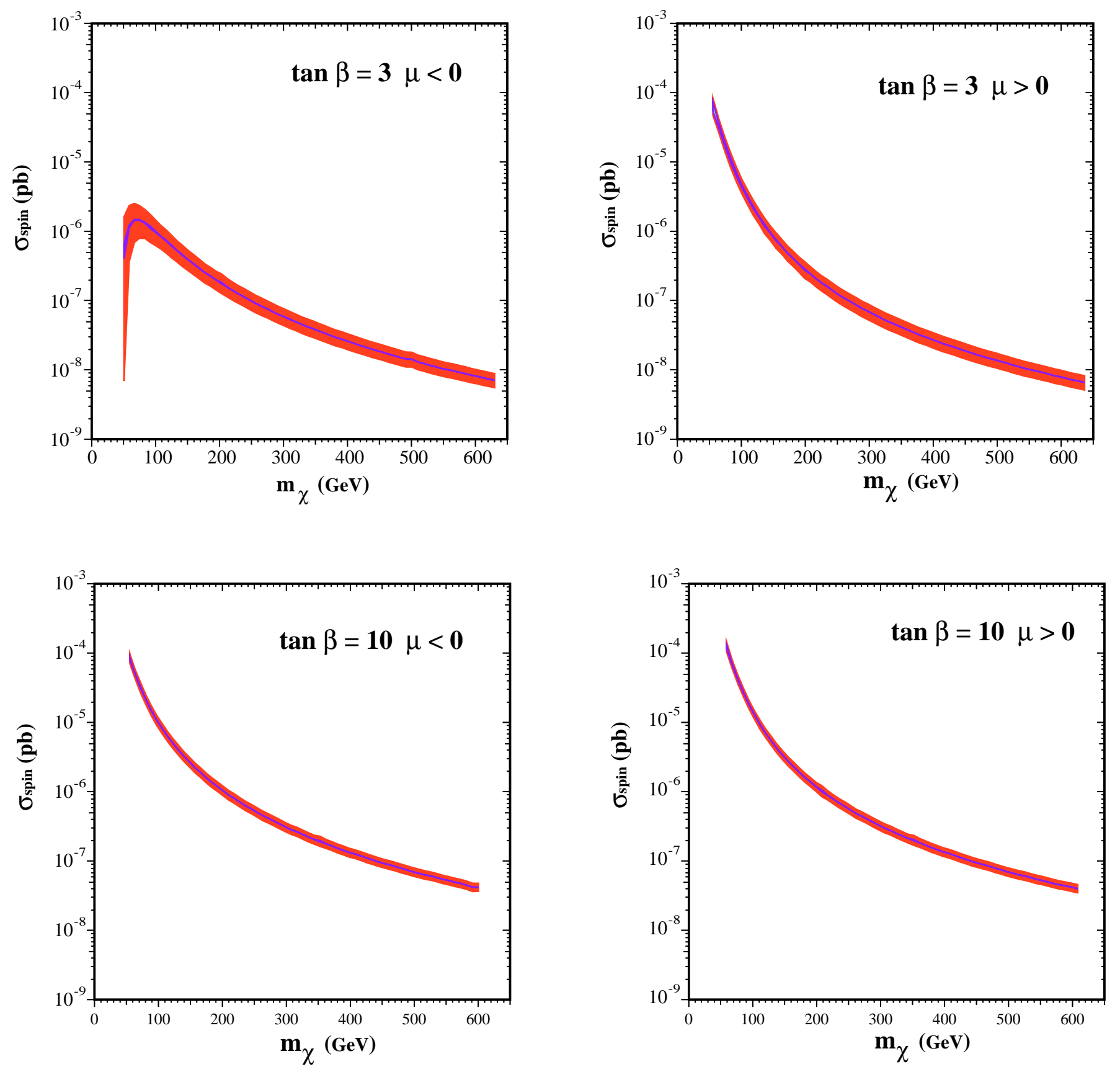

Figure 1: The spin-dependent cross section for the elastic scattering of neutralinos on protons as a function of the LSP mass. The central curves are based on the inputs (24), and their thicknesses are related to the spreads in the allowed values of $m_{0}$. The shaded regions correspond to the uncertainties in the hadronic inputs (24). 

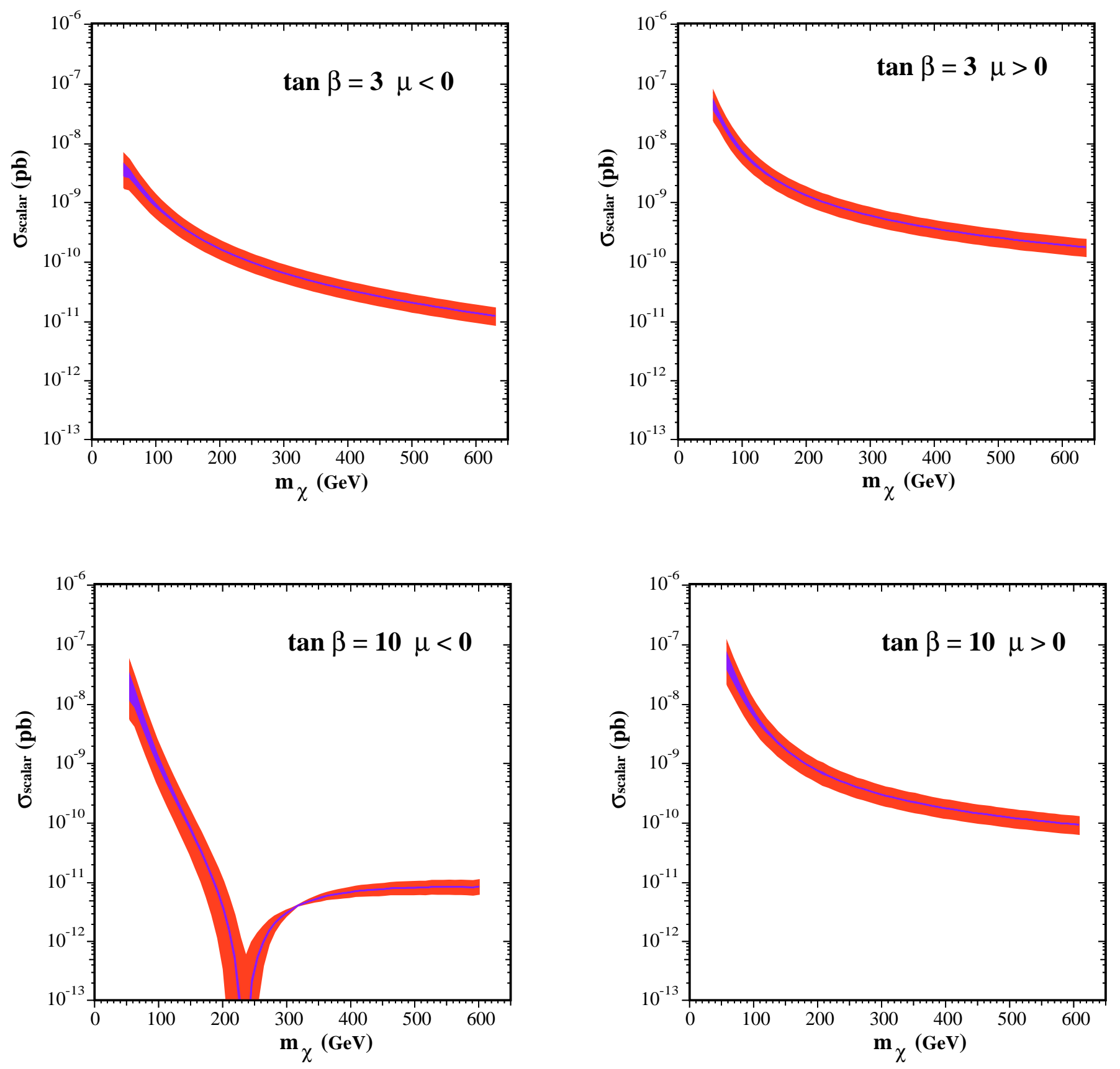

Figure 2: The spin-independent scalar cross section for the elastic scattering of neutralinos on protons as a function of the LSP mass. The central curves are based on the inputs (19), their thicknesses are again related to the spread in the allowed values of $m_{0}$, and the shaded regions now correspond to the uncertainties in the hadronic inputs (19). 
and protons are almost identical. Similarly, the effects of changing $A_{0}$ are also relatively small, as illustrated by two cases with $A_{0}=0$ in Figures $3 \mathrm{c}$ and $3 \mathrm{~d}$. In the latter example, there is almost a factor of 2 difference at higher values of $m_{\chi}$, which is due to yet another cancellation, this time between the squark-exchange and $Z$-exchange terms in $\alpha_{2 u}$.

Finally, we show in Figure 4 compilations of our results for the spin-dependent and -independent cross sections, compared with current and projected experimental limits obtained from [25. The shaded region in panel (a) is the union of the shaded regions in Figure 1 , and the shaded region in panel (b) is the union of the shaded regions in Figure 2.

\section{Discussion}

As seen in Figure 4, the present experimental upper limit 25 on the spin-independent part of the elastic scattering of the LSP on a nucleon is around $10^{-5}$ pb for $50 \mathrm{GeV} \lesssim m_{\chi} \lesssim 100 \mathrm{GeV}$. On the other hand, the maximum scalar cross section we find is around $10^{-8} \mathrm{pb}$, which is attained for $m_{\chi} \sim 50 \mathrm{GeV}$. This means that present experiments searching directly for supersymmetric dark matter are far from constraining the parameter space of our baseline theoretical framework, in which LEP constraints are applied to MSSM models with universal supergravity-inspired soft supersymmetry-breaking parameters $m_{1 / 2}, m_{0}$.

The literature contains predictions for the elastic LSP-nucleus scattering rates that vary considerably, with some estimates lying considerably higher than ours [6, 10]. There are various ways in which such differences might arise, of which we mention a few here. We have imposed the requirement that the LSP relic density lie in the favoured range $0.1 \leq$ $\Omega_{\chi} h^{2} \leq 0.3$, whereas other calculations often include models with lower relic densities. Such models would normally have larger $\chi \chi$ annihilation cross sections, and correspondingly larger elastic scattering cross sections. Hence the predicted scattering rates would be larger, if the conventional halo density $\rho \sim 0.3 \mathrm{GeV} / \mathrm{cm}^{3}$ is assumed for the LSP [25]. However, we believe this assumption is unreasonable: if not all the total cold dark matter density $\Omega_{C D M}$

is composed of LSPs, the density of LSPs in the halo should be reduced by the corresponding factor $\Omega_{\chi} / \Omega_{C D M}$.

Other possible differences may arise in the treatment of the LEP constraints: we find it to be almost excluded that the LSP be Higgsino-like [5], even if the assumptions of universal soft supersymmetry breaking are relaxed, and Higgsino dark matter is certainly excluded if universality is assumed, as is the case here. In addition to the LEP constraints, this is because the value of $\mu$ is predicted as a function of $m_{1 / 2}$ and $m_{0}$, placing the LSP firmly in the Bino-like region. The same considerations exclude an LSP with mixed Higgsino/gaugino 

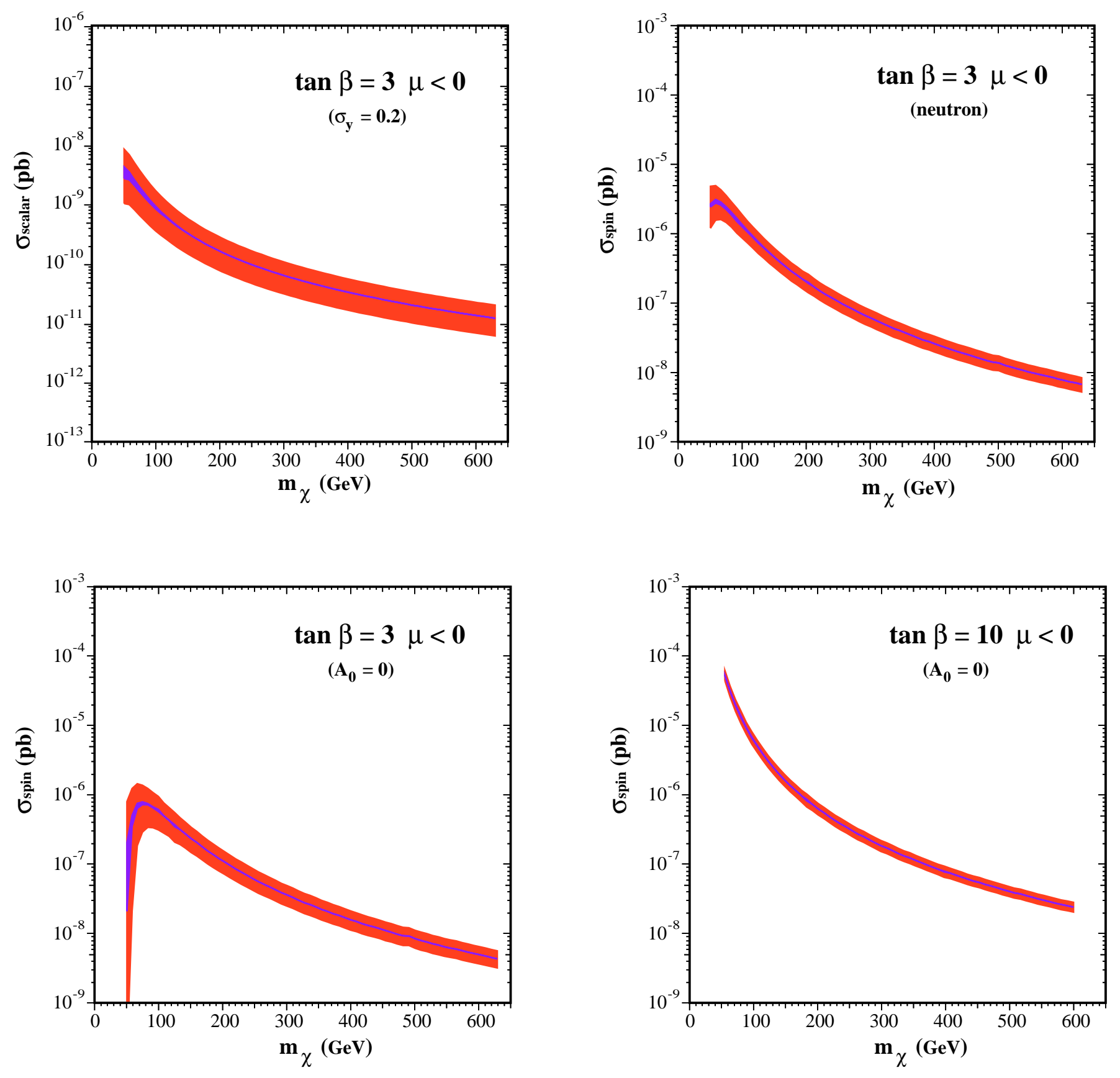

Figure 3: As in Figures 1 and 2, but now illustrating a) the effect of enlarging the uncertainty in $y$ to $\pm 0.2, b)$ the spin-dependent cross section for the elastic scattering of neutralinos on neutrons, c) and d) the effects of setting $A_{0}=0$ for $\mu<0$ and two choices of $\tan \beta$. 

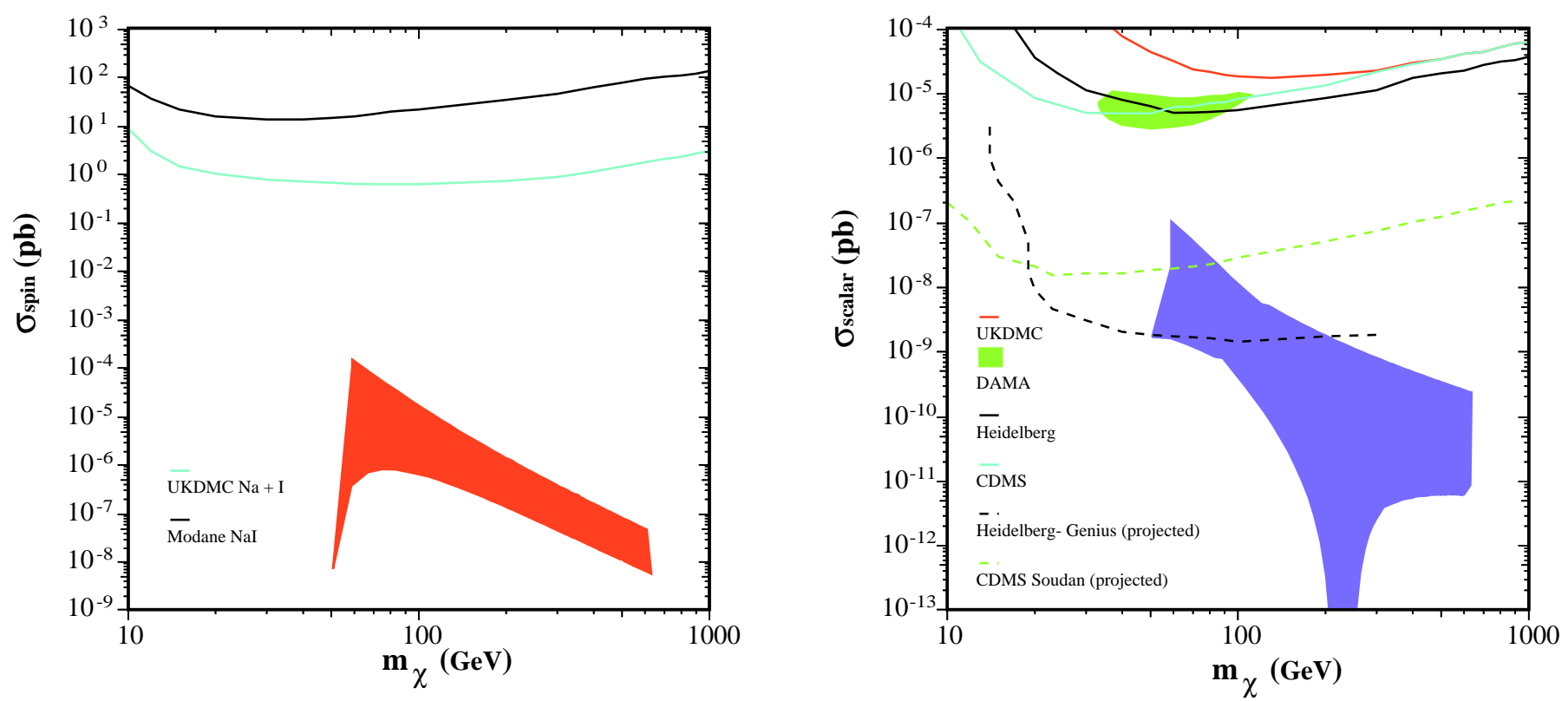

Figure 4: Summary plot compiling our predictions in the constrained MSSM for (a) the spin-dependent and (b) the spin-independent elastic LSP scattering cross sections on protons, compared in each case with current and projected limits on the scattering cross sections, as obtained from [25].

content.

The prediction of $\mu$ may be circumvented by postulating non-universality for the soft scalar supersymmetry-breaking parameters in the Higgs sector, which might have appeared to resurrect the possibility of a Higgsino-like LSP [26]. However, such a possibility goes beyond the universality framework adopted here, and, moreover, the LEP constraints now appear to exclude this possibility [5], as mentioned above.

There are no differences between the effective Lagrangians we and others [8, 16, 17, 18] use to describe the four-fermion $\chi-q$ interaction that determines the elastic $\chi-p, n$ scattering cross sections. We have found differences of detail between our and other treatments of the hadronic matrix elements of the scalar and axial-current $\bar{q} q$ operators appearing in this Lagrangian [27], but this is not responsible for any big difference between the results.

We should not want our experimental colleagues to be too downcast by the long road they appear to have to cover in order to probe the minimal universal MSSM framework utilized here. For example, there are surely some supersymmetric models that predict larger scattering rates. However, we think it best to have in mind a plausible and realistic target sensitivity, which is what our universal framework and implementation of the LEP and cosmological constraints provide. Our results also have the merit of being relatively specific: as seen in Figure 4, the elastic scattering cross sections we predict for any given value of the 
LSP mass $m_{\chi}$ lie in a comparatively narrow band. As discussed earlier, this is essentially because the LSP is always mainly Bino-like in our framework, so its couplings do not depend greatly on other MSSM parameters such as $m_{0}$. The principal causes of broadening are the uncertainties in the hadronic inputs and the possibilities of cancellations that may reduce the cross sections for some specific values of the constrained MSSM parameters.

This tight correlation we find between the LSP mass and its elastic scattering rate means that future experiments [28] should be able to phrase their sensitivities directly in terms of the LSP mass in the universal supergravity-inspired version of the MSSM. For example, our results suggest that the proposed Genius experiment 28] would be sensitive to $m_{\chi} \lesssim 100 \mathrm{GeV}$

for almost all MSSM parameter choices in Figure 5b. More optimistically, if/when a signal is observed, its plausibility would be enhanced if its recoil spectrum was correlated with the rate in the manner suggested by Figure 5. Thus our analysis provides experiments with an additional tool that may assist in the extraction of a signal that might be significantly smaller than they could have hoped. In any case, the importance of the search for supersymmetric matter remains unchanged, and there are still several years before the LHC comes into operation, so these experiments still have both motivation and opportunity.

\section{Acknowledgments}

We thank Toby Falk and Gerardo Ganis for many related discussions. The work of K.A.O. was supported in part by DOE grant DE-FG02-94ER-40823.

\section{References}

[1] J. Ellis, J.S. Hagelin, D.V. Nanopoulos, K.A. Olive and M. Srednicki, Nucl. Phys. B238 (1984) 453.

[2] For reviews, see: H.P. Nilles, Phys. Rep. 110 (1984) 1; H.E. Haber and G.L. Kane, Phys. Rep. 117 (1995) 75.

[3] L. Maiani, Proc. Summer School on Particle Physics, Gif-sur-Yvette, 1979 (IN2P3, Paris, 1980) p.3; G. 't Hooft, Recent Developments in Field Theory, eds. G. 't Hooft et al., (Plenum Press, New York, 1980); E. Witten, Nucl. Phys. B188 (1981) 513; R.K. Kaul, Phys. Lett. 109B (1982) 19.

[4] S. Dimopoulos, Phys. Lett. B246 (1990) 347. 
[5] J. Ellis, T. Falk, K.A. Olive and M. Schmitt, Phys. Lett. B388 (1996) 97 and Phys. Lett. B413 (1997) 355; J. Ellis, T. Falk, G. Ganis, K.A. Olive and M. Schmitt, Phys. Rev. D58 (1998) 095002.

[6] For a review, see, e.g., G. Jungman, M. Kamionkowski and K. Griest, Phys. Rep. 267 (1996) 195.

[7] M.W. Goldman and E. Witten, Phys. Rev. D31 (1986) 3059.

[8] J. Ellis and R. Flores, Nucl. Phys. B307 (1988) 883; Phys. Lett. B263 (1991) 259; Phys. Lett. B300 (1993) 175.

[9] K. Griest, Phys. Rev. D38 (1988) 2357; R. Barbieri, M. Frigeni and G. Giudice, Nucl. Phys. B313 (1989) 725; R. Flores, K.A. Olive and M. Srednicki, Phys. Lett. B237 (1990) 72; M. Drees and M. M. Nojiri, Phys. Rev. D48 (1993) 3483; V. Bednyakov, H.V. Klapdor-Kleingrothaus and S. Kovalenko, Phys. Rev. D50 (1994) 7128; H. Baer and M. Brhlik Phys. Rev. D57 (1998) 567.

[10] B. Sadoulet, Nucl. Phys. Proc. Suppl. 77 (1999) 389; A. Bottino, F. Donato, N. Forengo, and S. Scopel, Phys. Rev. D59 (1999) 095003; Phys. Rev. D59 (1999) 095004.

[11] H. Leutwyler, hep-ph/9609465.

[12] H.-Y. Cheng, Phys. Lett. B219 (1989) 347.

[13] J. Gasser, H. Leutwyler, and M. E. Sainio, Phys. Lett. B253 (1991) 252; M. Knecht, hep-ph/9912443.

[14] G. Mallot, hep-ex/9912040.

[15] J. Ellis, T. Falk and K.A. Olive, Phys. Lett. B444 (1998) 367; J. Ellis, T. Falk, K.A. Olive and M. Srednicki, hep-ph/9905481.

[16] T. Falk, A. Ferstl and K.A. Olive, Phys. Rev. D59 (1999) 055009.

[17] T. Falk, A. Ferstl and K.A. Olive, Astropart. Phys. (in press), hep-ph/9908311.

[18] U. Chattopadhyay, T. Ibrahim and P. Nath, Phys. Rev. D60 (1999) 063505.

[19] M. A. Shifman, A. I. Vainshtein and V. I. Zakharov, Phys. Lett. 78B, 443 (1978);

A. I. Vainshtein, V. I. Zakharov and M. A. Shifman, Usp. Fiz. Nauk 130, 537 (1980). 
[20] N. Bahcall, J. P. Ostriker, S. Perlmutter and P. J. Steinhardt, Science 284 (1999) 1481.

[21] The latest LEP limits on sparticles and Higgs bosons may be found in links on http://delphiwww.cern.ch/offline/physics_links/lepc.html.

[22] M. Carena, S. Heinemeyer, C.E. Wagner and G. Weiglein, hep-ph/9912223 and references therein.

[23] H. Baer, M. Brhlik and D. Castaño, Phys. Rev. D54 (1996) 6944; S. Abel and T. Falk, Phys. Lett. B444 (1998) 427.

[24] H. Baer and M. Brhlik, Phys. Rev. D55 (1997) 3201.

[25] R. Gaitskell and V. Mandic, http://cdms.berkeley.edu/limitplots/.

[26] V. Berezinsky, A. Bottino, J. Ellis, N. Fornengo, G. Mignola and S. Scopel, Astropart. Phys. 5 (1996) 1.

[27] A. Bottino, F. Donato, N. Fornengo and S. Scopel, hep-ph/9909228.

[28] L. Baudis et al., GENIUS Collaboration, hep-ph/9910205. 